Homology, Homotopy and Applications, vol.3(2), 2001, pp.355-359

\title{
IDEMPOTENTS AND LANDWEBER EXACTNESS IN BRAVE NEW ALGEBRA
}

\author{
J.P. MAY \\ (communicated by Gunnar Carlsson)
}

\begin{abstract}
We explain how idempotents in homotopy groups give rise to splittings of homotopy categories of modules over commutative $S$-algebras, and we observe that there are naturally occurring equivariant examples involving idempotents in Burnside rings. We then give a version of the Landweber exact functor theorem that applies to $M U$-modules.
\end{abstract}

In 1997, not long after [6] was written, I gave an April Fool's talk on how to prove that $B P$ is an $E_{\infty}$ ring spectrum or equivalently, in the language of [6], a commutative $S$-algebra. Unfortunately, the problem of whether or not $B P$ is an $E_{\infty}$ ring spectrum remains open. However, two interesting remarks emerged and will be presented here. One concerns splittings along idempotents and the other concerns the Landweber exact functor theorem.

One of the nicest things in $[6]$ is its one line proof that $K O$ and $K U$ are commutative $S$-algebras. This is an application of the following theorem [6, VIII.2.2], or rather the special case that follows.

Theorem 1. Let $R$ be a cell commutative $S$-algebra, $A$ be a cell commutative $R$ algebra, and $M$ be a cell $R$-module. Then the Bousfield localization $\lambda: A \longrightarrow A_{M}$ of $A$ at $M$ can be constructed as the inclusion of a subcomplex in a cell commutative $R$-algebra. In particular, the commutative $R$-algebra $A_{M}$ is a commutative $S$-algebra by neglect of structure.

The cell assumptions can always be arranged by use of the cofibrant replacement constructions in $[\mathbf{6}]$, so they result in no loss of generality. The theorem specializes as follows to algebraic localizations at elements of $R_{*}=\pi_{*}(R)$ [6, VIII.4.2].

Theorem 2. Let $R$ be a cell commutative $S$-algebra and $X$ a set of elements of $R_{*}$. The localization $\lambda: R \longrightarrow R\left[X^{-1}\right]$ that induces the algebraic localization $R_{*} \longrightarrow$ $R_{*}\left[X^{-1}\right]$ can be constructed as the unit of a cell commutative $R$-algebra.

The connective real $K$-theory spectrum $k o$ is a commutative $S$-algebra by multiplicative infinite loop space theory [11], and $K O$ is the localization $k o\left[\beta^{-1}\right]$ obtained

The author was partially supported by the NSF.

Received June 29, 2001, revised July 31, 2001; published on September 13, 2001.

2000 Mathematics Subject Classification: Primary 55N20, 55N91, 55P43

Key words and phrases: Brown-Peterson spectrum, Landweber exact functor theorem, complex cobordism, $E_{\text {infty }}$ ring spectrum.

(C) 2001, J.P. May. Permission to copy for private use granted. 
by inverting the Bott class. Therefore $K O$ is a commutative $k o$-algebra and thus a commutative $S$-algebra. That's the one line. Complex $K$-theory works similarly.

As a matter of algebra, idempotents give localizations. Since $M U$ arises in nature as an $E_{\infty}$ ring spectrum, that being the paradigmatic example that led to the definition [10], one might try to prove that $B P$ is a Bousfield localization of $M U$ and thus a commutative $M U$-algebra. That is April Fool's nonsense, but the basic idea has a correct version with other applications, as we shall explain. Essentially the same idea occurred independently to Schwänzl, Vogt, and Waldhausen, who gave quite different applications $[\mathbf{1 3}, \mathbf{1 4}]$.

Definition 3. Let $R$ be a cell commutative $S$-algebra and let $e \in R_{0}$ be an idempotent element. As a matter of algebra, $R_{*}\left[e^{-1}\right]=e R_{*}$. Define $e R$ to be the cell commutative $R$-algebra $R\left[e^{-1}\right]$ of Theorem 2 .

Theorem 4. Let $1=e_{1}+\cdots+e_{n}$ where the $e_{i}$ are orthogonal idempotents in $R_{*}$. Then the canonical map

$$
\varepsilon: R \longrightarrow e_{1} R \times \cdots \times e_{n} R
$$

of commutative $R$-algebras is a weak equivalence. Therefore the category of $R$ modules is Quillen equivalent to the product of the categories of $e_{i} R$-modules.

Proof. The first statement is obvious. The second statement follows from the next two results. The first is implicit in [6, III.4.2 and VII.4.8] and explicit in [9, I.3.6] and the second is proven by an easy formal argument.

Theorem 5. If $f: R \longrightarrow Q$ is a weak equivalence of commutative $S$-algebras, then the extension of scalars functor $f_{*}: \mathscr{M}_{R} \longrightarrow \mathscr{M}_{Q}$ and the pullback of structure functor $f^{*}: \mathscr{M}_{Q} \longrightarrow \mathscr{M}_{R}$ specify a Quillen equivalence of model categories.

Theorem 6. If $R$ is a product of commutative $S$-algebras $R_{i}$ with projections $\varepsilon_{i}$ : $R \longrightarrow R_{i}$, then the functor that sends an $R$-module $M$ to the tuple $\left(\varepsilon_{i *} M\right)$ is the left adjoint of a Quillen equivalence from $\mathscr{M}_{R}$ to the product of the categories $\mathscr{M}_{R_{i}}$. The right adjoint sends $\left(N_{i}\right)$ to the product of the $R$-modules $\varepsilon_{i}^{*} N_{i}$.

Theorem 4 shows that the homotopy theory of $R$-modules entirely decomposes into the homotopy theories of the modules over the $e_{i} R$. The ring spectra that algebraic topologists usually work with have no non-trivial idempotents. However, interesting examples do arise naturally in algebraic $K$-theory, as observed in [13].

Remark 7. If $R$ is connective, we have a map $R \longrightarrow H R_{0}$ that induces an isomorphism on $\pi_{0}$ [6, IV.3.1]. Here, if $X \subset R_{0}$ and we apply the functor $(-) \wedge_{R} H R_{0}$ to $\lambda: R \longrightarrow R\left[X^{-1}\right]$, we obtain a model for the localization

$$
\lambda: H R_{0} \cong R \wedge_{R} H R_{0} \longrightarrow R\left[X^{-1}\right] \wedge_{R} H R_{0} \cong\left(H R_{0}\right)\left[X^{-1}\right] \cong H\left(R_{0}\left[X^{-1}\right]\right) .
$$

In particular, for an idempotent $e \in R_{0}, e R \wedge_{R} H R_{0}$ is equivalent to $H\left(e R_{0}\right)$. This observation is the starting point of $[\mathbf{1 3}, \mathbf{1 4}]$.

Interesting examples also arise in equivariant algebraic topology. The results above generalize directly to the equivariant setting of commutative $S_{G}$-algebras 
and their modules $[\mathbf{6}, \mathbf{9}, \mathbf{1 2}]$, where $G$ is a compact Lie group and $S_{G}$ is the sphere $G$-spectrum. Here, for a commutative $S_{G}$-algebra $R$, we take $R_{*}=\pi_{*}\left(R^{G}\right)$. In particular, $\left(S_{G}\right)_{*}$ is the equivariant stable homotopy groups of spheres and $\left(S_{G}\right)_{0}$ is isomorphic to the Burnside ring $A(G)$. The ring $A(G)$, and more so its localizations at subrings of the rationals, usually does have non-trivial idempotents $[\mathbf{5}, \mathbf{8}]$.

The splittings of Theorem 4 give model theoretic refinements of splittings in equivariant stable homotopy theory that are discussed in $[\mathbf{8}, \mathrm{V}]$ and $[\mathbf{1 2}, \mathrm{XVII} \S 6]$. Those sources describe splittings of homology and cohomology theories, and it is now apparent that these splittings arise from splittings of corresponding equivariant stable categories. The splittings involve change of group functors, and these are discussed model theoretically in the contexts both of $S_{G}$-modules and of orthogonal $G$-spectra in [9]. Briefly, by [9, VI.1.2], for an inclusion $\iota: H \subset G$, there is a Quillen adjoint pair $\left(G_{+} \wedge_{H}(-), \iota^{*}\right)$ relating $H \mathscr{M}$ to $G \mathscr{M}$. Let $W H=N H / H$ and let $\varepsilon: N H \longrightarrow W H$ be the quotient homomorphism. By [9, 3.12], there is also a Quillen adjoint pair relating $N H \mathscr{M}$ to $W H \mathscr{M}$. This remains true after localization at a prime or rationalization. Thus we can split localized stable categories along idempotents and identify the pieces as equivalent to stable categories over subquotient groups.

We now turn to a completely different topic, but one that also arises naturally from consideration of spectra constructed from $M U$, namely the Landweber exact functor theorem. In fact, that result has the following more structured version in the category of $M U$-modules. We say that an $M U_{*}$-module $M_{*}$ is Landweber exact if, for each prime $p$, the set $\left\{v_{i} \mid i \geqslant 0\right\}$ is a regular sequence for $M_{*}$. Here $v_{0}=p$ and the $v_{i}$ for $i>0$ are indecomposable elements of degree $2 p^{i}-2$ with Chern numbers divisible by $p$.

Theorem 8. If $M_{*}$ is a Landweber exact $M U_{*}$-module, then there is an $M U$-module $M$ such that $\pi_{*}(M)=M_{*}$ and, for any finite cell $M U$-module $X$,

$$
\pi_{*}(X) \otimes_{M U_{*}} M_{*} \cong \pi_{*}\left(X \wedge_{M U} M\right) .
$$

As a matter of algebra, Landweber $[\mathbf{7}, 2.6]$ proved the following result. Let $\mathscr{M} \mathscr{U}$ denote the category of comodules over $M U_{*}(M U)$ that are finitely presented as $M U$-modules.

Theorem 9 (Landweber). The functor $(-) \otimes_{M U_{*}} M_{*}$ on the category $\mathscr{M} \mathscr{U}$ is exact if and only if the $M U_{*}$-module $M_{*}$ is Landweber exact.

By the following two results, $M U$-modules naturally gives rise to objects of $\mathscr{M} \mathscr{U}$.

Lemma 10. If $X$ is a finite cell $M U$-module, then $\pi_{*}(X)$ is a finitely presented $M U_{*}$-module.

Proof. This is proven by exactly the same induction on the number of cells as in the classical special case $X=M U \wedge Y$, where $Y$ is a finite CW spectrum. Of course, in that case $\pi_{*}(X)=M U_{*}(Y)$. For example, the proof is clear from the algebraic argument given by Adams [1, pp. 132-133].

Lemma 11. If $X$ is an $R$-module, where $R$ is a commutative $S$-algebra such that $R_{*} R$ is $R_{*}$-flat, then the Hurewicz map gives $X_{*}$ a structure of $R_{*} R$-comodule. 
Proof. This is proven by diagram chasing as in Adams [1]. It is the starting point of the development of an Adams spectral sequence in brave new algebra [3]. The main point is that

$$
R_{*} R \otimes_{R_{*}} X_{*} \cong \pi_{*}\left((R \wedge R) \wedge_{R} X\right) \cong \pi_{*}\left(R \wedge_{R} X\right) .
$$

Of course, this applies with $R=M U$. The previous three results imply the following conclusion.

Proposition 12. Let $M_{*}$ be a Landweber exact $M U_{*}$-module. Then the functor $\pi_{*}(X) \otimes_{M U_{*}} M_{*}$ specifies a homology theory on finite cell $M U$-modules $X$.

Applying Adams' variant [2] of Brown's representability theorem, which applies since $M U_{*}$ is countable [6, III.2.13], we obtain the $M U$-module $M$ promised in Theorem 8. The construction of $M$ is non-uniquely functorial: given a map $f_{*}$ : $M_{*} \longrightarrow N_{*}$ of Landweber exact $M U$-modules, there is a map $f: M \longrightarrow N$ of $M U$ modules that realizes $f_{*}$, but $f$ will not be unique unless the relevant $\lim ^{1}$ groups vanish.

Example 13. Recall that $K U_{*}=\mathbb{Z}\left[u, u^{-1}\right]$, where $\operatorname{deg}(u)=2$, and give it the $M U_{*}$-module structure specified by the ring homomorphism $M U_{*} \longrightarrow K U_{*}$ that sends $\left[M^{2 n}\right]$ to $T d\left(M^{2 n}\right) u^{n}$. We know by the methods of $[\mathbf{6}, \mathrm{V} \S 4]$ that $K U$ is an $M U$-module and in fact an $M U$-ring spectrum. There results an isomorphism

$$
\pi_{*}(X) \otimes_{M U_{*}} K U_{*} \longrightarrow K U_{*}(X)
$$

for finite cell $M U$-modules $X$. Alternatively, granting that there is a unique ring spectrum $K U$ with the cited homotopy groups, we can construct $K U$ as an $M U$ module by Theorem 8 and then show that it is an $M U$-ring spectrum by the methods of [6, V§4]. The resulting map $T d: M U \longrightarrow K U$ is a map of $M U$-ring spectra. The calculation of $T d_{*}$ in terms of the Todd genius is evident from the present approach, but is not clear from the approach of $[6, \mathrm{~V} \S 4]$. In any case, this gives a generalization to $M U$-modules of the Conner-Floyd theorem that $M U$-theory determines $K U$-theory.

Of course, if $B P$ is a commutative $S$-algebra, then the Landweber exact functor theorem will admit a precisely analogous and more useful version for $B P_{*}$-modules.

\section{References}

[1] J.F. Adams. Lectures on generalized cohomology. Springer Lecture Notes in Mathematics Vol 99, 1969, pp 1-138.

[2] J.F. Adams. A variant of E.H. Brown's representability theorem. Topology 10(1971), 185-198.

[3] A. Baker and A. Lazarev. On the Adams spectral sequence for $R$-modules. Preprint, 2001.

[4] P.E. Connor and E.E. Floyd. The relation of cobordism to $K$-theories. Springer Lecture Notes in Mathematics Vol. 28. 1966. 
[5] T. tom Dieck. Idempotent elements in the Burnside ring. J. Pure and Applied Algebra. 10(1977), 239-247.

[6] A. D. Elmendorf, I. Kriz, M. A. Mandell, and J. P. May (with an appendix by M. Cole). Rings, modules, and algebras in stable homotopy theory. Mathematical Surveys and Monographs Vol. 47. 1997. American Mathematical Society.

[7] P. S. Landweber. Homological properties of comodules over $M U_{*}(M U)$ and $B P_{*}(B P)$. American J. Math. 98(1976), 591-610.

[8] L. G. Lewis, J. P. May, and M. Steinberger, with contributions by J. E. McClure. Equivariant stable homotopy theory. Lecture Notes in Mathematics Vol. 1213. Springer-Verlag, 1986.

[9] M. A. Mandell and J.P. May Equivariant orthogonal spectra and $S$-modules. Memoirs Amer. Math. Soc. To appear.

[10] J. P. May (with contributions by F. Quinn, N. Ray, and J. Tornehave). $E_{\infty^{-}}$ ring spaces and $E_{\infty}$-ring spectra. Springer Lecture Notes in Mathematics Vol. 577. 1977.

[11] J.P. May. Multiplicative infinite loop space theory. J. Pure and Applied Algebra 26(1983), 1-69.

[12] J. P. May, et al. Equivariant homotopy and cohomology theory. CBMS Regional Conference Series in Mathematics, Number 91. American Mathematical Society. 1996.

[13] R. Schwänzl, R.M. Vogt, and F. Waldhausen. Adjoining roots of unity to $E_{\infty}$ ring spectra in good cases - a remark. in Homotopy invariant algebraic structures. Contemp. Math Vol. 239, 1999, 245-249. Amer. Math. Soc.

[14] R. Schwänzl, R.M. Vogt, and F. Waldhausen. Topological Hoschschild homology. J. London Math. Soc.(2) 62(2000), 345-356.

This article may be accessed via WWW at http://www.rmi.acnet.ge/hha/ or by anonymous ftp at

ftp://ftp.rmi.acnet.ge/pub/hha/volumes/2001/n2a4/v3n2a4.(dvi,ps,pdf)

J.P. May may@uchicago.edu

Department of Mathematics

University of Chicago

Chicago, IL 60637 